\title{
Type la SN progenitors: pre-explosion phase in nearly Chandrasekhar mass WDs
}

\author{
Inma Domínguez ${ }^{1, *}$, Luciano Piersanti ${ }^{2}$, Eduardo $\mathrm{Bravo}^{3}$, Oscar Straniero ${ }^{2}$, and Sergio \\ Cristallo ${ }^{2}$ \\ ${ }^{1}$ Universidad de Granada 18071, Granada, Spain \\ ${ }^{2}$ INAF-Osservatorio Astronomico d'Abruzzo, I-64100, Teramo, Italy \\ ${ }^{3}$ E.T.S. Arquitectura del Vallés, Universitat Politècnica de Catalunya, 08173 Sant Cugat del Vallès, \\ Spain
}

\begin{abstract}
Type Ia supernovae are used as distance indicators to measure the expansion rate of the Universe and to constrain the nature of dark energy. Current and upcoming surveys will allow to extend supernova Hubble diagrams to higher redshifts and to improve further their statistics.

It is accepted that Type Ia supernovae are thermonuclear explosions of carbonoxygen white dwarfs in binary systems. However, the identification of their progenitors, the evolutionary path leading to the explosion and the explosion mechanism itself have not been identified yet. This is critical, as we need to understand the potential evolution of their luminosity with cosmic time and, thus, with their stellar progenitors.

We will review the current situation, considering observational hints. We will focus on our recent models, that follow the evolution of carbon-oxygen white dwarfs accreting mass up to thermonuclear runaway, and on their dependence with the initial metallicity of the white dwarf progenitors.
\end{abstract}

\section{Introduction}

Bright and homogeneous, Type Ia Supernovae (SNeIa) are our best extragalactic distance indicators. Since the 60s SNIa Hubble diagrams (HDs) have been used to measure the value of the Hubble-Lemaitre constant, which is still a subject of hot debate [e.g. 1]. SNIa HDs show the recent acceleration of the expansion rate of the universe [2, 3], and its previous deceleration [4]. The challenge today is to reach enough precision in SNIa HDs to reveal the nature of that enigmatic component of the Universe, which currently dominates its dynamics, the dark energy [see 5]. SNeIa are not equal, and the precision of SNeIa as distance indicators relies on calibration relations, such as the maximum-decline relation [6], that link maximum absolute magnitudes with the shape of the light curve peaks and colours. New calibration relations are proposed trying to increase the precision [7], which in current HDs is above 0.15 mag. Moreover, SNeIa play a central role in the origin and evolution of the nuclei, in particular, of the so called iron group nuclei, being responsible of about $60 \%$ of their universal abundances [e.g. 8, 9].

\footnotetext{
*e-mail: inma@ugr.es
} 
In spite of their central role in cosmology, in nucleosynthesis, and in other fields, SNIa progenitors and explosion mechanisms are still unknown. The classical scenario consists of a thermonuclear explosion of a carbon-oxygen white dwarf (CO WD), which accreting mass from a companion reachs explosion conditions when its mass is very close to the Chandrasekhar mass limit [10], MCh scenario. Starting from C-ignition in the central inner zone, the whole WD is incinerated and its ashes contain intermediate-mass elements and a substantial amount of iron group nuclei. Among these nuclei, ${ }^{56} \mathrm{Ni}$ is mostly produced, and it is the radioactive energy from the cascade ${ }^{56} \mathrm{Ni}-{ }^{56} \mathrm{Co}-{ }^{56} \mathrm{Fe}$ that powers SNIa light curves (LCs). More ${ }^{56} \mathrm{Ni}$ implies more luminosity at maximum. This simple scenario perfectly agrees with SNIa homogenity and their tight calibration relation. It also implies that, to a great extent, the memory of the properties of the WD progenitor, such as the initial mass and chemical composition, is lost in the supernova outcomes, i.e., LCs and spectra.

At the other side, there is a growing amount of evidences pointing to a dependence of the LC properties with the local and/or general properties of the host galaxies. Brighter (postmaximum slow decline LCs) SNe Ia are associated with young stellar populations, in which the SNIa rate is higher. Dimmer (post-maximum fast decline LCs) also occur in galaxies in which star formation ceased several Gyr ago, and there, the SNIa rate is smaller. Moreover, after calibration, a dependence remains with the galaxy total stellar mass, and likely with specific star formation rate and metallicity, which may be interpreted as a dependence with the progenitor stellar population age [e.g. 11-19].

It may well be that two or more progenitor systems contribute to the normal (non peculiar) SNIa population. In fact, several progenitor systems have been proposed [see for detailed reviews, 20-23]. A critical property is the total mass of the WD at the explosion time, which is strictly connected to the explosion mechanism. Explosions of sub-Chandrasekhar mass WDs (sub-MCh) have been suggested to represent a significant part of SNe Ia. In these subMCh WDs, the explosion starts with a detonation at the external recently accreted He-rich layer. Then, an inward shock moves toward the center triggering a carbon detonation. Also, the nature of the companion object is still under debate: broadly speaking, it could be a normal star (Single Degenerate, or SD, scenario) or another WD (Double Degenerate, or DD, scenario). Other scenarios, that may explain some peculiar events, have been proposed, such as the merging of a $\mathrm{CO}$ WD with the $\mathrm{CO}$ core of an AGB companion star, or violent mergers of two white dwarfs.

\section{Selected hints from observations and implications}

Hints of a non-degenerate companion (MS, RG or AGB stars) have not been found yet. Hydrogen is not detected at maximum light epoch (otherwise it would be a Type II SNe) and, in most events, neither at late times, disfavouring $\mathrm{H}$ accretion. In the well studied nearby $\mathrm{SNe}, 2011 \mathrm{fe}$ and 2014J, a single degenerate companion has been excluded for $2011 \mathrm{fe}$ and a RG or an AGB companion for 2014J [24-27]. Late LCs are expected to show signatures of non-degenerate companions, but no detection has been claimed yet, as in the recent study [28] including above $100 \mathrm{SNe}$ Ia. Although these observations are challenge and more high quality data are needed, they are easily explained within the DD scenario. Moreover, delayed time distributions and rates agree better with the DD scenario [19, 22, 29].

At the other side, SNIa nebular spectra [30, 31] and some Supernova Remnants (like Tycho, Kepler, 3C 397 and N103B) observations [e.g. 32-36] require burning at high densities and thus, better agree with a MCh explosion.

Taking together, observations suggest that the MCh DD scenario plays a major role. But then, Why the outcome of thermonuclear explosions of CO WDs with the same mass (MCh) should be related with the stellar population from which they come from ? 


\section{Models, results and conclusions}

In this work we explore in detail the physical and chemical properties of the pre-explosive evolution of the MCh DD scenario. For all details we refer to our recent accepted article [37].

Based on the 1D hydrostatic code FUNS [38, 39], we follow the evolution of the WD progenitors, the accretion phase up to $\mathrm{C}$-ignition and the subsequent convective C-burning phase up to the explosion, which is assumed to occur when the temperature reaches 800 MK. We include e-captures and all the relevant URCA reactions. The initial model is the one presented in [40], which consists of two CO WDs with initial masses $0.6 \mathrm{M}_{\odot}$ and 0.8 $\mathrm{M}_{\odot}$ and $\mathrm{Z}=0.138$. As the WD increases its mass due to accretion, it contracts and its density increases, reaching the threshold densities of different e-capture nuclear reactions. At the corresponding URCA shell, the rate of electron captures and beta decays are equal, while in the denser zones, bellow the URCA shell, e-captures dominate and in the less dense zones, above the URCA shell, the corresponding URCA pair nuclei decay. At the URCA shell, all terms in the energy budget cancel each other, except those corresponding to the neutrino and antineutrino emission, so URCA pairs produce cooling at the shell.

As the WD continues to accrete mass and contract, successive URCA shells form, while those previously formed move outwards.

After C-gnition, the situation becomes more complex, convection develops carrying URCA nuclei up and down. In fact, if convective URCA produced a net heating or a net cooling has been an open question for a long time. To calculate properly the URCA energy budget all terms should be consider. In particular, in the high density inner zones, heating is produced due to the thermal energy of the captured electrons, while cooling is produced due to the absorbed thermal energy needed to move down the electrons.

In order to include properly all physical and chemical processes we decide to adopt the following set-up for the simulations: (1) full coupling of physical and chemical evolution, including burning and mixing; (2) the Ledoux criterium to define the border of the convective zones, as the chemical gradients at the URCA shells are relevant and play a critical role; (3) full nuclear network, at least up to ${ }^{32} \mathrm{P}$, including all relevant e-captures and URCA pairs.

In order to investigate the impact of different initial metallicities of the WD progenitors on the explosion ignition conditions, we adopt 5 different initial metallicities: $\mathrm{Z}=(0.0245,0.6,1.38,2$ and 4$) \times 10^{-2}$, and accrete $\mathrm{CO}$ rich matter according to the same procedure described in [40] at a rate of $=10^{-7} \mathrm{M}_{\odot} / \mathrm{yr}$.

In agreement with previous works [40-44] we obtain that electron captures and URCA pairs imply a net cooling of the inner region, C-ignition occurs at higher densities and both, the explosion density and the neutronization levels, are higher as compared with models that do not include URCA reactions.

We identify, and this is a new result with respect to previous calculations, the relevant role played by the ${ }^{21}(\mathrm{Ne}, \mathrm{F})$ URCA pair [45]. The inclusion of this pair, along with the correct URCA energy budget and the Ledoux criterium, stops the growth of the convective core, and hence, stops the inward circulation of nuclei and electrons, and cooling dominates. Thus, the cooling of this isolated core implies that a larger amount of nuclear energy is needed to trigger the explosion, hence, the amount of C-consumption is also larger, as well as the explosion density and neutronization level. As the amount of ${ }^{21} \mathrm{Ne}$ depends on $\mathrm{Z}$, the maximum mass attained by the convective core and the explosion density also depend on $\mathrm{Z}$, except for the lower $\mathrm{Z}$, in which the amount of initial ${ }^{21} \mathrm{Ne}$ is too low and does not play any significant role.

Our results show that:

(1) Convective core masses at the explosion depend on $\mathrm{Z}$, increasing from $0.12 \mathrm{M}_{\odot}$ to $0.24 \mathrm{M}_{\odot}$ as $\mathrm{Z}$ increases, for the studied models. Note that different convective core masses imply different chemical gradients. 
(2) Explosion densities increase with Z, from 5.02 to $6.3410^{9} \mathrm{~g} / \mathrm{cm}^{3}$. These high densities may be a hint of high accretion rates.

(3) Both, the central and average (over the convective core mass) neutronization levels at the explosion time also increase with $\mathrm{Z}$ with respect to the corresponding initial values. In principle, if the neutronization level of the inner zones of a SNIa could be deduced from the observed chemical pattern of neutronised elements, it would be possible to infer the initial metallicity of the WD progenitor:

$$
Z_{\text {pro }}=10.975 \times \eta_{\text {exp }}-1.094 \times 10^{-3}
$$

\section{References}

[1] E. Macaulay, R.C. Nichol, D. Bacon, D. Brout, T.M. Davis, B. Zhang, B.A. Bassett, D. Scolnic, A. Möller, C.B. D’Andrea et al., 486, 2184 (2019), 1811.02376

[2] A.G. Riess, A.V. Filippenko, P. Challis, A. Clocchiatti, A. Diercks, P.M. Garnavich, R.L. Gilliland, C.J. Hogan, S. Jha, R.P. Kirshner et al., 116, 1009 (1998), astro-ph/9805201

[3] S. Perlmutter, G. Aldering, G. Goldhaber, R.A. Knop, P. Nugent, P.G. Castro, S. Deustua, S. Fabbro, A. Goobar, D.E. Groom et al., 517, 565 (1999), astro-ph/9812133

[4] A.G. Riess, L.G. Strolger, J. Tonry, S. Casertano, H.C. Ferguson, B. Mobasher, P. Challis, A.V. Filippenko, S. Jha, W. Li et al., 607, 665 (2004), astro-ph/0402512

[5] M. Betoule, R. Kessler, J. Guy, J. Mosher, D. Hardin, R. Biswas, P. Astier, P. El-Hage, M. Konig, S. Kuhlmann et al., 568, A22 (2014), 1401 . 4064

[6] M.M. Phillips, 413, L105 (1993)

[7] C.R. Burns, M. Stritzinger, M.M. Phillips, E.Y. Hsiao, C. Contreras, S.E. Persson, G. Folatelli, L. Boldt, A. Campillay, S. Castellón et al., 789, 32 (2014), 1405 . 3934

[8] F.X. Timmes, S.E. Woosley, T.A. Weaver, 98, 617 (1995), astro-ph/9411003

[9] N. Prantzos, C. Abia, M. Limongi, A. Chieffi, S. Cristallo, 476, 3432 (2018), 1802.02824

[10] F. Hoyle, W.A. Fowler, 132, 565 (1960)

[11] M. Hamuy, M.M. Phillips, N.B. Suntzeff, R.A. Schommer, J. Maza, R. Aviles, 112, 2398 (1996), astro-ph/9609062

[12] M. Hamuy, S.C. Trager, P.A. Pinto, M.M. Phillips, R.A. Schommer, V. Ivanov, N.B. Suntzeff, 120, 1479 (2000), astro-ph/0005213

[13] F. Mannucci, M. Della Valle, N. Panagia, E. Cappellaro, G. Cresci, R. Maiolino, A. Petrosian, M. Turatto, 433, 807 (2005), astro-ph/0411450

[14] M. Sullivan, D. Le Borgne, C.J. Pritchet, A. Hodsman, J.D. Neill, D.A. Howell, R.G. Carlberg, P. Astier, E. Aubourg, D. Balam et al., 648, 868 (2006), astro-ph/0605455

[15] Y. Kang, Y.W. Lee, Y.L. Kim, C. Chung, C.H. Ree, 889, 8 (2020), 1912.04903

[16] L. Kelsey, M. Sullivan, M. Smith, P. Wiseman, D. Brout, T.M. Davis, C. Frohmaier, L. Galbany, M. Grayling, C.P. Gutiérrez et al., 501, 4861 (2021), 2008. 12101

[17] M.E. Moreno-Raya, Á.R. López-Sánchez, M. Mollá, L. Galbany, J.M. Vílchez, A. Carnero, 462, 1281 (2016), 1607.05526

[18] M. Rigault, Y. Copin, G. Aldering, P. Antilogus, C. Aragon, S. Bailey, C. Baltay, S. Bongard, C. Buton, A. Canto et al., 612, C1 (2018)

[19] P. Wiseman, M. Sullivan, M. Smith, C. Frohmaier, M. Vincenzi, O. Graur, B. Popovic, P. Armstrong, D. Brout, T.M. Davis et al., arXiv e-prints arXiv:2105.11954 (2021), 2105. 11954 
[20] D. Branch, J.C. Wheeler, Supernova Explosions (2017)

[21] B. Wang, Z. Han, 56, 122 (2012), 1204.1155

[22] D. Maoz, F. Mannucci, G. Nelemans, 52, 107 (2014), 1312.0628

[23] M. Livio, P. Mazzali, 736, 1 (2018), 1802.03125

[24] W. Li, J.S. Bloom, P. Podsiadlowski, A.A. Miller, S.B. Cenko, S.W. Jha, M. Sullivan, D.A. Howell, P.E. Nugent, N.R. Butler et al., 480, 348 (2011), 1109. 1593

[25] F. Patat, M.A. Cordiner, N.L.J. Cox, R.I. Anderson, A. Harutyunyan, R. Kotak, L. Palaversa, V. Stanishev, L. Tomasella, S. Benetti et al., 549, A62 (2013), 1112 . 0247

[26] P.L. Kelly, O.D. Fox, A.V. Filippenko, S.B. Cenko, L. Prato, G. Schaefer, K.J. Shen, W. Zheng, M.L. Graham, B.E. Tucker, 790, 3 (2014), 1403.4250

[27] R. Margutti, J. Parrent, A. Kamble, A.M. Soderberg, R.J. Foley, D. Milisavljevic, M.R. Drout, R. Kirshner, 790, 52 (2014), 1405.1488

[28] M.A. Tucker, B.J. Shappee, P.J. Vallely, K.Z. Stanek, J.L. Prieto, J. Botyanszki, C.S. Kochanek, J.P. Anderson, J. Brown, L. Galbany et al., 493, 1044 (2020), 1903.05115

[29] A. Castrillo, Y. Ascasibar, L. Galbany, S.F. Sánchez, C. Badenes, J.P. Anderson, H. Kuncarayakti, J.D. Lyman, A.I. Díaz, 501, 3122 (2021), 2012 . 11958

[30] C.L. Gerardy, W.P.S. Meikle, R. Kotak, P. Höflich, D. Farrah, A.V. Filippenko, R.J. Foley, P. Lundqvist, S. Mattila, M. Pozzo et al., 661, 995 (2007), astro-ph/0702117

[31] C.M. Telesco, P. Höflich, D. Li, C. Álvarez, C.M. Wright, P.J. Barnes, S. Fernández, J.H. Hough, N.A. Levenson, N. Mariñas et al., 798, 93 (2015), 1409. 2125

[32] C. Badenes, E. Bravo, J.P. Hughes, 680, L33 (2008), 0805. 3344

[33] S. Park, C. Badenes, K. Mori, R. Kaida, E. Bravo, A. Schenck, K.A. Eriksen, J.P. Hughes, P.O. Slane, D.N. Burrows et al., 767, L10 (2013), 1302 . 5435

[34] H. Yamaguchi, C. Badenes, A.R. Foster, E. Bravo, B.J. Williams, K. Maeda, M. Nobukawa, K.A. Eriksen, N.S. Brickhouse, R. Petre et al., 801, L31 (2015), 1502.04255

[35] H. Martínez-Rodríguez, C. Badenes, S.H. Lee, D.J. Patnaude, A.R. Foster, H. Yamaguchi, K. Auchettl, E. Bravo, P.O. Slane, A.L. Piro et al., 865, 151 (2018), 1807.02990

[36] Y. Ohshiro, H. Yamaguchi, S.C. Leung, K. Nomoto, T. Sato, T. Tanaka, H. Okon, R. Fisher, R. Petre, B.J. Williams, 913, L34 (2021), 2105 . 04101

[37] L. Piersanti, E. Bravo, O. Straniero, S. Cristallo, I. Dominguez, arXiv e-prints arXiv:2112.02943 (2021), 2112.02943

[38] O. Straniero, R. Gallino, S. Cristallo, Nuclear Physics A 777, 311 (2006), astro-ph/0501405

[39] S. Cristallo, O. Straniero, R. Gallino, L. Piersanti, I. Domínguez, M.T. Lederer, 696, 797 (2009), 0902.0243

[40] L. Piersanti, E. Bravo, S. Cristallo, I. Domínguez, O. Straniero, A. Tornambé, G. Martínez-Pinedo, 836, L9 (2017), 1701.06453

[41] D.A. Chamulak, E.F. Brown, F.X. Timmes, K. Dupczak, 677, 160-168 (2008), 0801.1643

[42] A.L. Piro, L. Bildsten, 673, 1009-1013 (2008), 0710. 1600

[43] H. Martínez-Rodríguez, A.L. Piro, J. Schwab, C. Badenes, 825, 57 (2016), 1602 . 00673

[44] J. Schwab, H. Martínez-Rodríguez, A.L. Piro, C. Badenes, 851, 105 (2017), 1711.04780

[45] J. Iben, I., 226, 996 (1978) 